\title{
Paracoccidioidomicose aguda/subaguda disseminada. Primeiro caso no Rio Grande do Sul
}

\author{
Acute/subacute disseminated paracoccidioidomycosis. \\ First case in Rio Grande do Sul State, Brazil
}

Soraya Malafaia Colares, Silvana Marcantônio, Simone Zambonato e Luiz Carlos Severo

\begin{abstract}
Resumo É relatado o primeiro caso autóctone de paracoccidioidomicose disseminada aguda/subaguda ocorrido em criança no Rio Grande do Sul. A doença iniciou com adenomegalias superficiais generalizadas, seis meses antes da internação hospitalar. O diagnóstico foi feito através de biópsia de gânglio cervical. É comentado o espectro de formas clínicas da micose observado nesse Estado.
\end{abstract}

Palavras-chaves: Paracoccidioidomicose. Micose sistêmica em criança. Rio Grande do Sul.

Abstract The first autochthonous case of acute/subacute disseminated paracoccidioidomycosis observed in a child in Rio Grande do Sul (Brazil) is reported. The disease started with widespread superficial lymphadenopathy six months before the patient was admitted to the hospital. The diagnosis was made through a cervical lymph node biopsy. The spectrum of the clinical forms of the mycosis observed in this State is commented upon.

Key-words: Paracoccidioidomycosis. Systemic mycosis in children. Rio Grande do Sul.

Hospital da Criança Santo Antônio e Instituto de pesquisas e Diagnóstico (IPD), Santa Casa de Misericórdia, Porto Alegre, RS. Endereço para correspondência: Dr. L.C. Severo. IPD-Santa Casa, Annes Dias 285, 90020-090 Porto Alegre, RS, Brasil. Fax: (051) 214-8435.

E-mail: severo@santacasa.tche.br

Recebido para publicação em 13/01/98 
Campos, em 19423, relatou os dois primeiros casos de paracoccidioidomicose (PCM) observados no Rio Grande do Sul. Um dos pacientes, jovem sul-rio-grandense de 22 anos, viera doente do Rio de Janeiro, onde residia, e onde provavelmente adquirira a infecção. O paciente apresentava a forma disseminada subaguda da micose, tipo juvenil. Esta forma clínica da micose ainda não foi descrita em nativos e residentes no estado.

A presente publicação tem dois objetivos. Primeiro, relatar o primeiro caso autóctone de PCM disseminada subaguda que é, ao mesmo tempo, o primeiro dessa forma clínica em criança sul-rio-grandense. Em segundo lugar, comentar sobre a gama de formas clínicas da PCM observadas no Rio Grande do Sul.

\section{RELATO DO CASO}

Em 21/02/92, menina parda, de 9 anos, natural e procedente da zona rural (pais lavradores) de Tapes, RS, internou em nosso hospital por emagrecimento e adenomegalias superficiais generalizadas, com 6 meses de duração. Há duas semanas apresentava febre $\left(39^{\circ} \mathrm{C}\right)$. Ao exame físico observou-se desnutrição protéico-calórica de terceiro grau $(18 \mathrm{~kg})$; linfonodos das cadeias cervicais, supraclaviculares, retroauriculares, occipitais, submentonianas, pré-auriculares, axilares e inguinais aumentados de volume, alguns endurecidos, outros amolecidos; fígado palpável a $2 \mathrm{~cm}$ da reborda costal. O exame histopatológico de biópsia de gânglio cervical e o exame direto e cultivo do pus deste linfonodo permitiram o diagnóstico de paracoccidioidomicose. Imunodifusão revelou banda de precipitação específica. Exames bacteriológicos (microscopia de esfregaços corados ao Gram e ZiehlNeelsen; cultivos em meios de ágar sangue e Loewenstein-Jensen) do pus do linfonodo não revelaram bactérias. Reação de Mantoux foi negativa. A paciente foi tratada com sulfametoxazol-trimetoprim, deixando o hospital para acompanhamento ambulatorial.

Em maio de 1994, voltou ao hospital com o mesmo quadro clínico. Usara o sulfamídico irregularmente e suspendera seu uso. Foi reiniciada a sulfamidoterapia e a paciente deixou o hospital em junho.

A terceira internação ocorreu em 24/11/95. Queixava-se de dor abdominal, diarréia, disúria e edema dos membros inferiores. Entre os dados laboratoriais mais importantes: leucopenia
(2900 leucócitos $/ \mathrm{mm}^{3}$ ), albumina sérica $=$ $1,6 \mathrm{mg} / \mathrm{dl}$ e proteínas $=3,6 \mathrm{mg} / \mathrm{dl}$; anticorpos antiHIV, pela técnica de ELISA: negativo; ao hemocultivo foi isolado Escherichia coli. Radiograma de tórax mostrou opacidades com atelectasia e consolidações focais no pulmão direito (Figura 1); calcificações em linfonodos da cadeia cervical esquerda e supraclavicular. Tomografia abdominal evidenciou acentuada hepatomegalia com nítida diminuição da densidade do órgão; pâncreas sem anormalidades; presença de adenomegalias pré-aórticas, algumas calcificadas; lesão com densidade de partes moles e com parte central de menor densidade, medindo cerca de $2,5 \mathrm{~cm}$ de diâmetro e com localização retroperitoneal à direita, na altura da pelve renal, em situação paraórtica e retrocava, provavelmente linfadenomegalia com necrose (Figura 2). Enema opaco revelou acentuada distensão de alças intestinais, estenose inflamatória em região do sigmóide, com marcada redução de calibre, contornos irregulares, espessamento das pregas e presença de ulcerações; extenso comprometimento do cólon ascendente e do ceco por espessamento das pregas da mucosa, redução de calibre, irregularidade de contornos por edema e lesões inflamatórias de mucosa (Figura 3). Passou a receber cetoconazol, deixando o hospital melhorada e em controle ambulatorial.

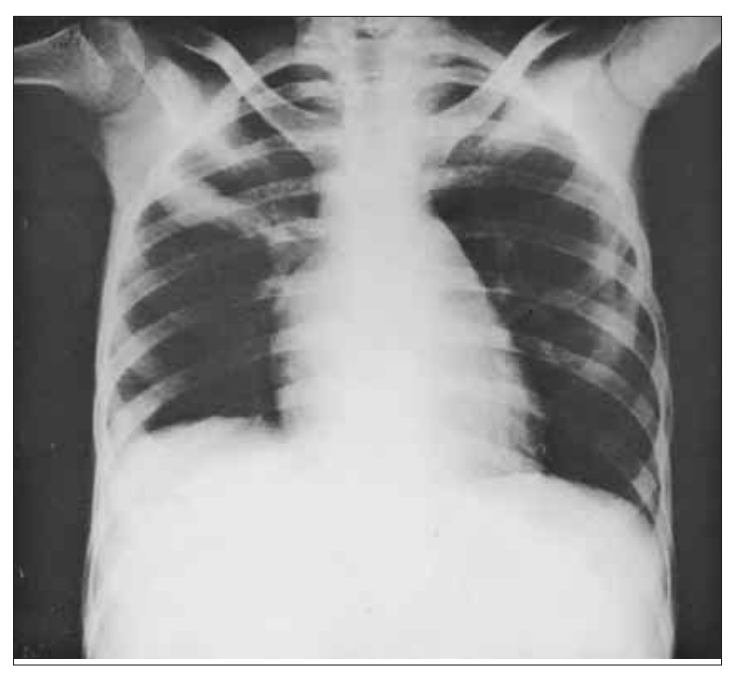

Figura 1 - Radiograma em frontal do tórax demonstrando área de infiltração com atelectasia subsegmentar no lobo superior díreito e mínimas áreas de consolidações no terço inferior desse pulmão. 


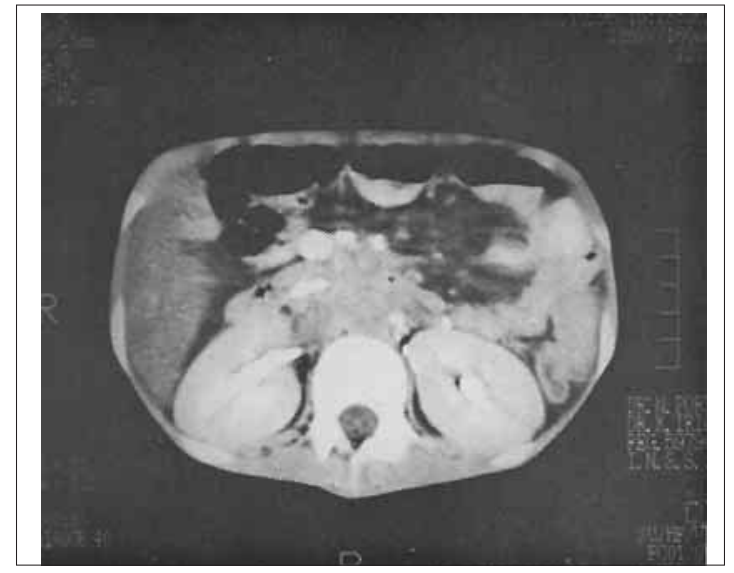

Figura 2 - Tomografia computadorizada com contraste de abdômen. Ao nível do hilo renal revela importante comprometimento ganglionar retroperitoneal.
A quarta internação ocorreu em 16/11/96. A paciente apresentava dor abdominal intensa e vômitos. Foi iniciada a administração de anfotericina B. Radiograma contrastado do esôfago, estômago e duodeno evidenciou úlceras no delgado. Radiografia simples do abdômen revelou distensão de alças intestinais (Figura 4). Enema opaco mostrou distensão de alças, sem ar no reto, barro biliar, aumento do volume do fígado e ascite. A paciente foi submetida a laparostomia que demonstrou ileíte, por isso foi executada ileostomia. Por insuficiência respiratória e necessidade de ventilação mecânica a doente foi transferida para UTI e passou a receber fluconazol intravenoso ao esquema antifúngico. A seguir a paciente apresentou sepse por Staphylococcus aureus, falência de múltiplos órgãos e morte.

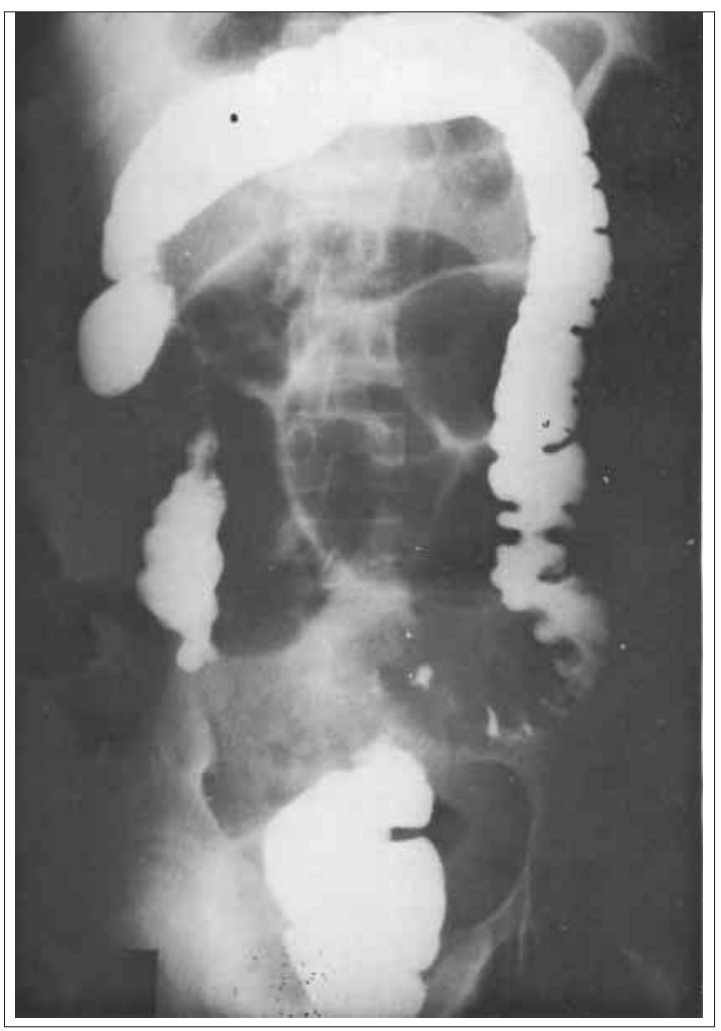

Figura 3 - Enema opaco demonstrando incompetência da válvula íleo-cecal com possível estenose do íleo terminal.

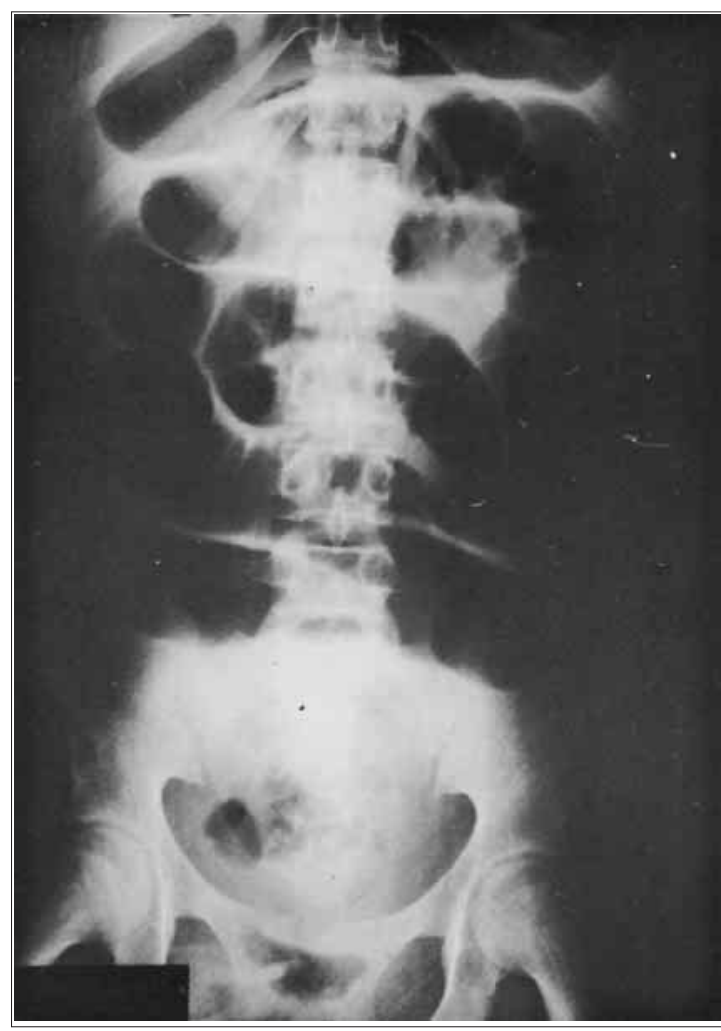

Figura 4 - Radiograma simples de abdômen demonstrando importante distensão gasosa de alças intestinais, especialmente do intestino delgado. 


\section{DISCUSSÃO}

A classificação das formas clínicas da PCM, caráter de progressividade e gravidade de tipo clínico, seguida foi a apresentada por Wanke e Londero24. Com exceção da forma disseminada aguda/subaguda (tipo juvenil) da PCM, as demais formas clínicas da micose foram observadas e relatadas em indivíduos nativos e residentes do Rio Grande do Sul. A forma subclínica (infecção) foi evidenciada pela prova intradérmica com paracoccidioidina25; foram relatados casos de complexo linfo pulmonar19, de forma pulmonar primária regressiva ${ }^{13}$, de forma pulmonar primária progressiva (aguda) ${ }^{7}$, de lesões pulmonares residuais 1117 22, de forma pulmonar crônica8 16 18, de forma disseminada crônica8 18 e de forma oportunística uni20 21 ou multifocal1 10. O presente caso - forma aguda/subaguda disseminada completa o espectro de formas clínicas da PCM no estado.

O presente caso é, também, o terceiro caso de PCM em crianças no Rio Grande do Sul, porém, com uma particularidade: os dois primeiros relatam manifestações resultantes de lesões circunscritas ao pulmão7 13. Os três provinham de área endêmica da micose ${ }^{2}$, encosta do planalto 713 e vertente do maciço cristalino, presente caso. Além disso, é importante salientar que no Uruguai (país vizinho), fisiográfica e economicamente semelhante ao RS, não foi descrito a micose em crianças5.

A paciente apresentou a modalidade mais comum da forma aguda/subaguda disseminada - a modalidade linfática9. Da mesma maneira, como nos casos tratados com sulfamídicos, em geral irregularmente, houve recaída com manifestações mais graves que as da apresentação inicial23.
Recente revisão sobre a PCM na criança brasileira conseguiu coligir 269 casos da doença. Porém, somente em 77 (28,6 \%) desses casos são relatados dados suficientes para analisar o início e os sinais e sintomas mais proeminentes da apresentação da micose, que é polimórfica, Setenta e quatro desses pacientes apresentavam a forma aguda/subaguda disseminada e, desses, 24 (34,4\%), a modalidade linfática 9 . As lesões pulmonares, como observado no presente caso, deveriam ser mais freqüentes do que o assinalado na literatura, porém o estudo deste órgão tem sido negligenciado9.

A PCM disseminada aguda/subaguda da criança tem curso imprevisível. As recaídas são freqüentes - 26,7\% de 30 casos de uma série 23 .

A PCM não deve fugir à regra das demais micoses sistêmicas causadas por fungos termodimórficos patogênicos. A infecção paracoccidióidica ocorre desde a infância6 12 e casos da doença foram registrados em crianças de 3 anos de idade15. A PCM deve apresentarse na criança e no jovem sob a forma de infecção pulmonar primária sintomática, de curso regressivo ou progressivo. Quando de curso progressivo, costuma ser o início de forma disseminada aguda/subaguda4 14. A PCM merece que sejam definidas claramente as manifestações clínicas que ocorrem no jovem e na criança.

\section{AGRADECIMENTOS}

Ao Dr. Alberto Thomaz Londero (Professor Emérito, Universidade Federal de Santa Maria, Santa Maria, RS), pelas críticas e sugestões.

\section{REFERÊNCIAS BIBLIOGRÁFICAS}

1. Bakos L, Kronfeld M, Hampe S, Castro I, Zampese M. Disseminated paracoccidioidomycosis with skin lesions in a patient with acquired immunodeficiency syndrome (letter). Journal of Academy of Dermatology 20:854-855, 1989.

2. Bopp C, Bernardi CDU. Geopatologia da blastomicose sul-americana no Rio Grande do Sul. Revista da Associação Médica do Rio Grande do Sul 11:31-49, 1967.

3. Campos EC. Sobre dois casos de granuloma paracoccidióidico no Rio Grande do Sul. Arquivos do Departamento Estadual de Saúde do Rio Grande do Sul 3:71-77, 1942.
4. Campos EP, Bertoli CJ, Barbosa KS. Linfonodo pulmonar na paracoccidioidomicose aguda infantil (Relato de caso). Revista da Sociedade Brasileira de Medicina Tropical 25:195-200, 1992.

5. Conti-Díaz IA. Aspectos epidemiológicos y geográficos regionales de la blastomicosis sudamericana. El Tórax 17:35-39, 1968.

6. Freitas NA. Paracoccidioidomicose. Inquérito com a paracoccidioidina e busca ativa de casos no município de Campos, RJ. Tese de Mestrado. Universidade Federal do Rio de Janeiro, Rio de Janeiro, RJ, 1992.

7. Londero, AT. Paracoccidioidomicose. I. Patogenia, formas clínicas, manifestações pulmonares e diagnóstico. Jornal de Pneumologia 12:41-60, 1986. 
8. Londero AT, Ramos CD. Paracoccidioidomicose. Estudo clínico e micológico de 260 casos observados no interior do Estado do Rio Grande do Sul. Jornal de Pneumologia 16:129-132, 1990.

9. Londero AT, Rios-Gonçalves AJ, Terra GMF, Nogueira SA. Paracoccidioidomycosis in brazilian children. A critical review (1911-1994). Arquivos Brasileiros de Medicina 70:197-203, 1996.

10. Londero AT, Santos JW, Silva LA, Ramos CD. Paracoccidioidomicose associada a droga imunosupressora em paciente com lupus eritematoso sistêmico. Jornal de Pneumologia 13:224-226, 1987.

11. Melo IS, Londero AT. Spontaneously resolving pulmonary lesions in paracoccidioidomycosis. Mycopathologia 82:57-59, 1983.

12. Pedrosa PN. Paracoccidioidomicose: inquérito intradérmico com paracoccidoidina em zona rural do Estado do Rio de Janeiro. Dissertação de Mestrado, Universidade Federal do Rio de Janeiro, Rio de Janeiro, RJ, 1976.

13. Ramos CD, Londero AT, Gal MCL. Pulmonary paracoccidioidomycosis in a nine year old girl. Mycopathologia 74:15-18, 1981.

14. Rios-Gonçalves AJ, Rosembaum R, Londero AT, Terra GMF, Carvalho LM, Borges VM. Apresentação incomum da paracoccidioidomicose disseminada "tipo juvenil". Arquivos Brasileiros de Medicina 66:335-337, 1992.

15. Rios-Gonçalves AJ, Terra GMF, Rosembaum R, Nogueira SA, Mesquita CC, Londero AT. Paracoccidioidomicose em crianças de três anos de idade. Apresentação de dois casos. Arquivos Brasileiros de Medicina 70:73-79. 1996.

16. Santos JWA. Estudo das lesões pulmonares exclusivas na paracoccidioidomicose. Contribuição ao ao seu estudo no Rio Grande do Sul. Dissertação de Mestrado. Universidade Federal do Rio Grande do Sul, Porto Alegre, RS,1995.
17. Santos JWA, Michel GT, Londero AT. Paracoccidioidoma: case record and review. Mycopathologia 137:83-85, 1997.

18. Severo LC. Paracoccidioidomicose. Estudo clínico e radiológico das lesões pulmonares e seu diagnóstico. Tese de Mestrado, Universidade Federal do Rio Grande do Sul, Porto Alegre, RS, 1979.

19. Severo LC, Geyer GR, Londero AT, Porto NS, Rizzon CFC. The primary pulmonary lymph node complex in paracoccidioidomycosis. Mycopathologia 67:115118, 1979.

20. Severo LC, Londero AT, Geyer GR, Porto NS. Acute pulmonary paracoccidioidomycosis in an immunosuppressed patient. Mycopathologia 68:171174, 1979.

21. Severo LC, Palombini BC, Utz E, Braun SN Paracoccidioidomicose pulmonar resultante de reativação de lesão quiescente, em paciente imunossuprimido. Jornal de Pneumologia 6:21-22, 1980.

22. Severo LC, Porto NS, Camargo JJ, Geyer GR. Multiple paracoccidioidomas simulating Wegener's granulomatosis. Mycopathologia 91:117-119, 1985.

23. Terra GMF. Contribuição ao estudo clínico da paracoccidioidomicose na criança. Tese de Mestrado, Universidade Federal do Rio de Janeiro, Rio de Janeiro, RJ, 1993.

24. Wanke B, Londero AT. Paracoccidioides brasiliensis. In: Collier L, Bllows A, Sussman M (eds) Topley \& Wilson's microbiology and microbial infections. 9th edition, Arnold, London, p. 395-407, 1998.

25. Zembrzuski MM, Bassanesi MC, Wagner LC, Severo LC. Inquérito com histoplasmina e paracoccidioidina em duas regiões do Rio Grande do Sul. Revista da Sociedade Brasileira de Medicina Tropical 28:1-3, 1996. 